\title{
Treating allergic bronchopulmonary aspergillosis: the way forward
}

\author{
Richard B. Moss \\ Affiliation: Center for Excellence in Pulmonary Biology, Dept of Pediatrics, Stanford University School of \\ Medicine, Stanford, CA, USA. \\ Correspondence: Richard B. Moss, Center for Excellence in Pulmonary Biology, Dept of Pediatrics, Stanford \\ University School of Medicine, 770 Welch Road, Palo Alto, CA 94034, USA. E-mail: rmossdastanford.edu
}

0 $@$ ERSpublications

ABPA remains a difficult disease to control and entails troubling steroid toxicity http://ow.ly/V4mrR

Allergic bronchopulmonary mycoses are an endotype of allergic lung disease, similar to but more severe than uncomplicated asthma, caused by a Th2-dominated immune response and a bronchocentric granulocytic inflammation provoked by endobronchial growth of filamentous fungi in individuals with innate host defence defects (primarily people with severe allergic asthma or cystic fibrosis); untreated, it leads to progressive structural damage [1-3]. The scope of this problem, however, has only been recognised recently $[4,5]$.

In $>90 \%$ of cases, Aspergillus fumigatus is the causative fungal agent; allergic bronchopulmonary aspergillosis (ABPA) has been recognised as a distinct clinical entity since the 1950s [6]. For over 35 years, months-long courses of oral glucocorticosteroids (OGCS) have been the mainstay of ABPA treatment, based on early uncontrolled studies demonstrating near-universal clinical, radiographic and immunological responses [7-9]. However, no controlled studies have ever been performed on this primary treatment modality, and so dose and duration of OGCS courses have been left to clinical trial-and-error and accumulated experience [10].

For reasons that remain unclear, ABPA appears to be more prevalent in south Asia than in many other regions of the world [11]; recently, investigators led by Ritesh Agarwal of the Postgraduate Institute of Medicine Education and Research in Chandigarh, India, who care for a uniquely large cohort of people with ABPA, employed a OGCS regime with higher doses and longer exposures than that commonly used, hoping to lengthen the duration of the remission stage of ABPA [6, 12]. Now, AgARwAL et al. [13] have completed a randomised, controlled, open-label trial of two OGCS regimes in ABPA, as reported in this issue of the European Respiratory Journal.

Beyond praise for conducting one of the few controlled trials published on ABPA treatment, several cautionary aspects of this trial merit comment. First, only new, treatment-naïve cases presenting with acute ABPA were studied, so the applicability to relapsed or chronic (steroid-dependent) ABPA is unclear. Second, concomitant therapy with other agents potentially active in ABPA was excluded (more on this topic shortly). Third, collection of data on adverse effects of OGCS was included but not comprehensive in scope. Fourth, only ABPA in asthmatics was studied, so results cannot necessarily be applied to cystic fibrosis. Finally, selection criteria restrict the overall generalisability of the study, as does the underpowering of the sample size, driven by both study subject attrition and design overestimation of the treatment effect difference between the two OGCS regimes, which was derived from two prior studies of the prevalence of steroid-dependent ABPA that were published 20 years apart from centres on different continents. Nevertheless, the main finding, that the efficacy of the lower dose regime was similar to that of the higher dose regime, while steroid side-effects were lessened, is important, in that harm from overtreatment of ABPA can be reduced based on the results of this study. The lower dose regime employed by AgARWAL et al. [13] is very similar to that widely employed in Europe, North America and elsewhere.

Received: Nov 032015 | Accepted: Nov 122015

Conflict of interest: Disclosures can be found alongside the online version of this article at erj.ersjournals.com

Copyright @ERS 2016 
This study raises the important question of how further progress in treating ABPA is to occur, given the large number of case reports and series but paucity of controlled trials. First, better case ascertainment is needed, particularly in patients with asthma who are often managed without an appropriate index of suspicion for ABPA risk [14]. As diagnosis remains a complex process [6, 15], a simpler diagnostic approach remains an unmet need. Recently, an A. fumigatus-specific basophil activation assay has been found to be a robust diagnostic test in ABPA associated with cystic fibrosis, but it requires specialised flow cytometry expertise and expedited processing, as well as further study of utility in asthmatic ABPA [16, 17]. Even with improved diagnostic methods, pooling of patients via multicentre studies seems a needed component to improve feasibility as well as generalisability of controlled trials.

Other approaches to ABPA treatment to complement or replace OGCS have been the subject of increasing interest and scrutiny but more controlled trials are clearly needed. Can monthly high-dose "pulse" intravenous GCS further reduce steroid toxicity while maintaining or even improving efficacy over conventional OGCS $[18,19]$ ?

What about antifungal therapy? The use of oral antifungal azole agents with high activity against A. fumigatus has gained widespread use in ABPA and is recommended as second-line treatment in both asthma and cystic fibrosis $[6,15]$. However, reviews have emphasised the weakness of the evidence for safety and efficacy of azoles, with only two small, short-term, randomised, double-blind, placebo-controlled trials in asthmatic ABPA, and none in cystic fibrosis ABPA [20,21]. Can azole monotherapy be considered a viable alternative to OGCS? How long should azoles be used? In what stage(s) of ABPA? Is therapeutic drug monitoring necessary? How dangerous are cytochrome P450 3A4-mediated drug-drug interactions, particularly with certain systemic and inhaled glucocorticosteroid agents [22-24]? How ominous is the emerging problem of Aspergillus azole resistance [25, 26]? Fortunately, some of these issues are now being addressed in ongoing controlled trials of ABPA azole treatment by the Chandigarh group. One randomised, open-label trial is comparing OGCS to oral itraconazole monotherapy (www.clinicaltrials.gov identifier number NCT01321827), another OGCS to oral voriconazole (NCT 01621321) and a third OGCS monotherapy to combination OGCS-itraconazole therapy (NCT0244009). These studies should greatly contribute to understanding the proper role of azoles in ABPA.

Are there potential alternative approaches to antifungal treatment that avoid systemic effects, azole resistance and drug-drug interactions? Inhaled amphotericin B has been explored as an ABPA treatment with varying results in uncontrolled studies [27-29]. The lack of controlled trials is also being tackled in a Chandigarh study comparing nebulised amphotericin B deoxycholate combined with an inhaled GCS to inhaled GCS monotherapy in the maintenance of ABPA remission (NCT01857479); a randomised, single-blind, controlled trial of nebulised liposomal amphotericin B in maintaining remission is also underway in France (NCT02273661).

Finally, the success of omalizumab (anti-IgE monoclonal antibody) in improving control of moderate-severe allergic asthma has led to great interest and rapidly increasing usage in ABPA, usually undertaken as a steroid-sparing agent, with virtually unanimous reporting of reduced steroid requirements and exacerbations in published uncontrolled studies [30-33]. However, a recent Cochrane review rightly concluded that omalizumab use cannot be comfortably recommended in the absence of valid controlled trial data [34]. Unfortunately, an industry-sponsored, multicentre attempt at a double-blind, placebo-controlled, randomised trial in cystic fibrosis APBA (NCT00787917) was terminated early due to enrolment and retention issues, almost certainly related to a preposterously unrealistic dose regime (600 mg injected daily for 6 months). Actually, real-world omalizumab dosing in ABPA can be addressed by using a dose calculation resulting in a near-conventional treatment regime [31]. Accordingly, a randomised, double-blind, placebo-controlled omalizumab trial in adult asthmatics with ABPA using a cross-over design and dose regime of $750 \mathrm{mg}$ monthly was recently published, validating the uncontrolled literature: omalizumab works [35]. Validation of similar efficacy in a cystic fibrosis ABPA controlled trial is warranted.

Due to its complexity of diagnosis and treatment, ABPA remains a difficult disease to control and entails troubling steroid toxicity. As is common in medicine, adventures in alternatives have long peppered the scene, with an expected publication bias towards favourable results and weak standards of evidence, and so the solidity of well-designed controlled trials is still where, as Willie Sutton said of banks, you need to go to get the money.

\section{References}

1 Lötvall J, Akdis CA, Bacharier LB, et al. Asthma endotypes: a new approach to classification of disease entities within the asthma syndrome. J Allergy Clin Immunol 2011; 127: 355-360.

2 Becker KL, Gresnigt MS, Smeekens SP, et al. Pattern recognition pathways leading to a Th2 cytokine bias in allergic bronchopulmonary aspergillosis patients. Clin Exp Allergy 2015; 45: 423-437. 
3 Knutsen AP, Bush RK, Demain JG, et al. Fungi and allergic lower respiratory diseases. J Allergy Clin Immunol 2012; 129: 280-291.

4 Denning DW, Pleuvry A, Cole DC. Global burden of allergic bronchopulmonary aspergillosis with asthma and its complication chronic pulmonary aspergillosis in adults. Med Mycol 2013; 51: 361-370.

5 Armstead J, Morris J, Denning DW. Multi-country estimate of different manifestations of aspergillosis in cystic fibrosis. PLoS One 2014; 9: e98502.

6 Agarwal R, Chakrabarti A, Shah A, et al. Allergic bronchopulmonary aspergillosis: review of literature and proposal of new diagnostic and classification criteria. Clin Exp Allergy 2013; 43: 850-873.

7 Imbeau SA, Nichols D, Flaherty D, et al. Relationships between prednisone therapy, disease activity, and the total serum IgE level in allergic bronchopulmonary aspergillosis. J Allergy Clin Immunol 1978; 62: 91-95.

8 Wang JL, Patterson R, Roberts M, et al. The management of allergic bronchopulmonary aspergillosis. Am Rev Respir Dis 1979; 120: 87-92.

9 Capewell S, Chapman BJ, Alexander F, et al. Corticosteroid treatment and prognosis in pulmonary eosinophilia. Thorax 1989; 44: 925-929.

10 Moss RB. Critique of trials in allergic bronchopulmonary aspergillosis and fungal allergy. Med Mycol 2006; 44: S269-S272.

11 Agarwal R. Burden and distinctive character of allergic bronchopulmonary aspergillosis in India. Mycopathologia 2014; 178: 447-456.

12 Agarwal R, Gupta D, Aggarwal AN, et al. Allergic bronchopulmonary aspergillosis: lessons from 126 patients attending a chest clinic in north India. Chest 2006; 130: 442-448.

13 Agarwal R, Aggarwal AN, Dhooria S, et al. A randomised trial of glucocorticoids in acute-stage allergic bronchopulmonary aspergillosis complicating asthma. Eur Respir J 2016; 47: 490-498.

14 Greenberger PA, Bush RK, Demain JG, et al. Allergic bronchopulmonary aspergillosis. J Allergy Clin Immunol Pract 2014; 2: 703-708.

15 Stevens DA, Moss RB, Kurup VP, et al. Allergic bronchopulmonary aspergillosis in cystic fibrosis - state of the art. Clin Infect Dis 2003; 37: Suppl. 3, S225-S264.

16 Gernez Y, Dunn CE, Everson C, et al. Blood basophils from cystic fibrosis patients with allergic bronchopulmonary aspergillosis are primed and hyper-responsive to stimulation by aspergillus allergens. $J$ Cyst Fibros 2012; 11: 502-510.

17 Gernez Y, Walters J, Mirković B, et al. Blood basophil activation is a reliable biomarker of allergic bronchopulmonary aspergillosis in cystic fibrosis. Eur Respir J 2016; 47: 177-185.

18 Cohen-Cymberknoh M, Blau H, Shoseyov D, et al. Intravenous monthly pulse methylprednisolone treatment for ABPA in patients with cystic fibrosis. J Cyst Fibros 2009; 8: 253-257.

19 Sehgal IS, Agarwal R. Methylprednisolone pulse therapy is safe and effective for ABPA exacerbations unresponsive to conventional therapy. Eur Respir J 2014; 23: 149-152.

20 Moreira AS, Silva D, Ferreira AR, et al. Antifungal treatment in allergic bronchopulmonary aspergillosis with and without cystic fibrosis: a systematic review. Clin Exp Allergy 2014; 44: 1210-1227.

21 Elphick HE, Southern KW. Antifungal therapies for allergic bronchopulmonary aspergillosis in people with cystic fibrosis. Cochrane Database Syst Rev 2014; 11: CD002204.

22 Lebrun-Vignes B, Archer VC, Diquet B, et al. Effect of itraconazole on the pharmacokinetics of prednisolone and methylprednisolone and cortisol secretion in healthy subjects. Br J Clin Pharmacol 2001; 51: 443-450.

23 Bolland MJ, Bagg W, Thomas MG, et al. Cushing's syndrome due to interaction between inhaled corticosteroids and itraconazole. Ann Pharmacother 2004; 38: 46-49.

24 Gilchrist FJ, Cox KJ, Rowe R, et al. Itraconazole and inhaled fluticasone causing hypothalamic-pituitary-adrenal axis suppression in adults with cystic fibrosis. J Cyst Fibros 2013; 12: 399-402.

25 Chowdhary A, Sharma C, Hagen F, et al. Exploring azole antifungal drug resistance in Aspergillus fumigatus with special reference to resistance mechanisms. Future Microbiol 2014; 9: 697-711.

26 Verweij PE, Chowdhary A, Melchers WJ, et al. Azole resistance in Aspergillus fumigatus: can we retain the clinical use of mold-active antifungal azoles? Clin Infect Dis 2015 [In press DOI: 10.1093/cid/civ885].

27 Sehgal IS, Agarwal R. Role of inhaled amphotericin in allergic bronchopulmonary aspergillosis. J Postgrad Med 2014; 60: 41-45.

28 Chishimba L, Langridge P, Powell G, et al. Efficacy and safety of nebulised amphotericin B (NAB) in severe asthma with fungal sensitisation (SAFS) and allergic bronchopulmonary aspergillosis (ABPA). J Asthma 2015; 52: 289-295.

29 Casciaro R, Naselli A, Cresta F, et al. Role of nebulized amphotericin B in the management of allergic bronchopulmonary aspergillosis in cystic fibrosis: Case report and review of literature. J Chemother 2015; 27: $307-311$.

30 Moss RB. The use of biological agents for the treatment of fungal asthma and allergic bronchopulmonary aspergillosis. Ann NY Acad Sci 2012; 1272: 49-57.

31 Moss RB. Treatment options in several fungal asthma and allergic bronchopulmonary aspergillosis. Eur Respir J 2014; 43: 1487-1500

32 Lehmann S, Pfannenstiel C, Friedrichs F, et al. Omalizumab: a new treatment option for allergic bronchopulmonary aspergillosis in patients with cystic fibrosis. Ther Adv Respir Dis 2014; 8: 141-149.

33 Tanou K, Zintzaras E, Kaditis AG. Omalizumab therapy for allergic bronchopulmonary aspergillosis in children with cystic fibrosis: a synthesis of published evidence. Pediatr Pulmonol 2014; 49: 503-507.

34 Jat JR, Walia DK, Khairwa A. Anti-IgE therapy for allergic bronchopulmonary aspergillosis in people with cystic fibrosis. Cochrane Database Syst Rev 2013; 9: CD010288.

35 Voskamp AL, Gillman A, Symons K, et al. Clinical efficacy and immunologic effects of omalizumab in allergic bronchopulmonary aspergillosis. J Allergy Clin Immunol Pract 2015; 3: 192-199. 